\title{
ALGUNOS APUNTES SOBRE ENERGÍA Y TRIBUTACIÓN
}

\section{Taxation and energy: some considerations}

\author{
Beatriz de la Vega Rengifo* \\ Pontificia Universidad Católica del Perú
}

* Abogada por la Pontificia Universidad Católica del Perú (PUCP). LLM en Energía, Medio Ambiente \& Recursos Naturales en la Escuela de Derecho de la Universidad de Houston, Texas (Estados Unidos), LLM en Derecho de Hidrocarburos en la Universidad de Aberdeen, Escocia (Reino Unido), MBA por la Universidad Adolfo Ibáñez (Escuela de Negocios). Profesora de Tributación Minera e Hidrocarburos en la Facultad de Derecho de la PUCP. ORCID iD: 0000-0001-7389-9350. Contacto: bettydelavega@hotmail.com 


\title{
Resumen:
}

La energía es pilar fundamental para el desarrollo de la sociedad y los sectores económicos que la componen; sin embargo, es una de las mayores fuentes de emisión de Gases de Efecto Invernadero (GEI), por lo que múltiples países alrededor del globo han visto por conveniente promover cambios en su matriz energética, a través de incentivos tributarios a la inversión, generación y consumo de energía proveniente de fuentes menos contaminantes, como son los Recursos Energéticos Renovables (RER), e implementar a su vez impuestos al carbono, entre otras razones, para reducir el consumo de combustibles fósiles.

Todo ello se enmarca dentro de la lucha mundial contra el cambio climático y el cumplimiento de los compromisos asumidos en el marco del Acuerdo de París para reducir las emisiones de GEI a nivel país.

A continuación, se analiza cómo se ha venido dando la introducción de los impuestos al carbono en el contexto internacional ofreciendo una contextualización de los cambios realizados en múltiples países, no sin antes repasar algunos incentivos fiscales a la generación de energía mediante RER en el Perú, para finalmente reflexionar sobre la necesidad de que, además de una reforma regulatoria, se analice la incorporación de una reforma fiscal que considere el estatus de la transición energética y la lucha contra el cambio climático en el Perú sobre la base de la experiencia internacional previamente analizada; todo ello con el objetivo de ofrecer un profundo análisis sobre la evolución de los sistemas fiscales en la implementación de tributos relacionados con el sector de la energía y el cuidado del medio ambiente.

\begin{abstract}
:
Energy is a fundamental element for the development of society and the economic sectors that comprise it. However, it is one of the largest sources of Greenhouse Gas (GHG) emissions, which is why many countries around the globe have seen fit to promote changes in their energy matrix through tax incentives for investment, generation and consumption from less polluting energy sources such as Renewable Energy Resources (RER) and implementation of carbon taxes, among others, to reduce the consumption of fossil fuels.
\end{abstract}

All these actions are part of the global efforts against climate change and the fulfillment of the commitments assumed by all countries that signed the Paris Agreement to reduce GHG emissions at the country level.

In this sense, the introduction of carbon taxes in the international context 
is analyzed in the following document, offering a contextualization of the changes made in multiple countries, but not without first reviewing some tax incentives on power generation through RER in Peru. Finally, this document includes some comments regarding the need to evaluate a fiscal reform that considers the status of the energy transition and the fight against climate change in Peru. For these purposes, it is added an overview of the international experience with the aim of offering an in-depth analysis of the tax systems evolution about the implementation of taxes related to the energy sector and environmental care.

Palabras clave:

Impuestos - Energía - Recursos energéticos renovables - Impuesto al carbono - Reforma fiscal

Keywords:

Taxes - Energy - Renewable energy resources - Carbon tax - Tax reform

\section{Sumario:}

1. Introducción - 2. Aprovechamiento de Recursos Energéticos Renovables (RER) en el Perú - 3. Breves comentarios sobre el sector energético peruano, la generación mediante RER y sus incentivos - 4. Incentivos fiscales para energías renovables en el mundo - 5. El impuesto al carbono - 6. Reforma fiscal medioambiental en el Perú - 7. Conclusiones - 8 . Lista de referencias.

\section{INTRODUCCIÓN}

En la actualidad, una de las tendencias globales de mayor preocupación es el cambio climático, por lo que múltiples países alrededor del mundo se encuentran implementando políticas para reducir sus emisiones de gases de efecto invernadero.

Uno de los objetivos principales sería alcanzar el cumplimiento de los compromisos ambientales establecidos en el marco de la suscripción del Acuerdo de París, mediante la implementación de sus Contribuciones Nacionalmente Determinadas (CND) y las diversas Acciones Nacionales Apropiadas de Mitigación (NAMAS) que cada país habría diseñado e implementado.

Respecto a los compromisos ambientales es importante recordar que, en 1994, entró en vigencia la Convención Marco de las Naciones Unidas sobre 
el Cambio Climático (CMNUCC) mediante la cual se han formulado objetivos para mitigar la emisión de gases de efecto invernadero (GEI) para todos los países firmantes.

Posteriormente, en 1997, se firma el protocolo de Kyoto, el cual fue el primer intento de materializar las preocupaciones, objetivos y compromisos de los países firmantes de la CMNUCC mediante la definición de obligaciones concretas para la mitigación de GEI; sin embargo, no todos los Estados que fueran parte confirmaron su adhesión a dicho protocolo ${ }^{1}$.

Así, en el año 2007, con motivo de la Conferencia de las Partes de la CMNUCC (COP 13) y la correspondiente aprobación de la hoja de ruta de Bali, se acordó, entre otros, que los países en desarrollo llevarían a cabo NAMAS, con la finalidad de alcanzar el desarrollo sostenible, financiamiento y construcción de capacidades, en una forma medible, reportable y verificable.

En el año 2015, después de superar varios inconvenientes respecto de la brecha existente entre el volumen de emisiones de los países emergentes comparado con el de los países más desarrollados², se aprobaría el Acuerdo de París en la COP 21. Este último acuerdo sería uno de carácter histórico por el que los países miembros, en mérito a una causa común, emprenderían ambiciosos esfuerzos para combatir el cambio climático.

Es en ese contexto que, para el caso específico del sector energético, se plantea, entre otros puntos, la descarbonización del sector. Muchos países apuntan a llevar sus sectores energéticos a la neutralidad en carbono con miras a las próximas décadas, asumiendo nuevas tecnologías en la industria y políticas impositivas innovadoras. Ello, debido a que, a escala global y al cierre de 2018, el sector electricidad y producción de calor fue el que mayores emisiones tuvo, llegando a la cifra de 13,977.8 millones de toneladas métricas de $\mathrm{CO}_{2}\left(\mathrm{MMTCO}_{2}\right)$ y representando aproximadamente $35.85 \%$ del total de emisiones, seguido por

1 Si bien el Protocolo de Kioto fue firmado en 1997, no fue sino hasta 2005 que entró en vigor tras la ratificación de Rusia. Antes de aquella fecha, el Protocolo no cumplía con las condiciones para su puesta en marcha: era necesario que 55 países industrializados lo firmaran. Mediante este instrumento se acordó una reducción de al menos un $5 \%$ de las emisiones de gases de efecto invernadero (GEI) entre 2008 y 2012 en comparación con las emisiones de 1990.

2 Antes de la aprobación del Acuerdo de París, existía una división binaria entre países desarrollados y países en desarrollo respecto de su responsabilidad en la emisión de GEl, debido al alegato por parte del grupo de países desarrollados que únicamente estos estarían asumiendo responsabilidad por la emisión de GEl y acciones concretas para la reducción del calentamiento global, por lo que desde la celebración de la COP 15 en Copenhague (2009) se exigió mayor paridad entre este grupo y el de los países en desarrollo; situación que se fue corrigiendo y retomando cauce en las COP 16 a 20. 
el sector transporte que representa el $21 \%\left(6,158.3 \mathrm{MMTCO}_{2}\right)$, y la manufactura y construcción $\left(8,257.7 \mathrm{MMTCO}_{2}\right)$ que suponen el $15.75 \%{ }^{3}$.

La transición energética juega un papel sumamente importante para el cumplimiento de los compromisos ambientales de los países, debido a que la descarbonización del sector se efectuará de manera gradual con un rápido crecimiento de los Recursos Energéticos Renovables (RER) en el consumo de energía (3.1\% anual aproximadamente), pero todavía con una participación significativa de los combustibles fósiles al 2050, según las proyecciones de la Agencia de Información de Energía de los Estados Unidos- U.S. Energy Information Agency (EIA) (2019).

\section{Consumo de energía primaria global por tipo de energía(2010-2050)}

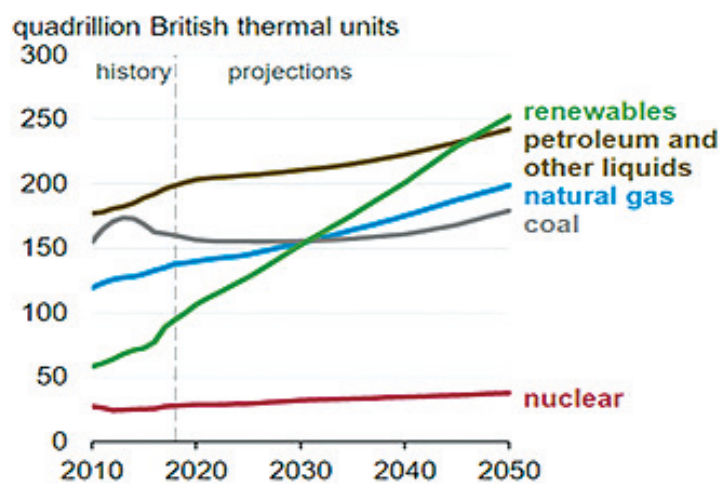

quadrillion British thermal units

1,000

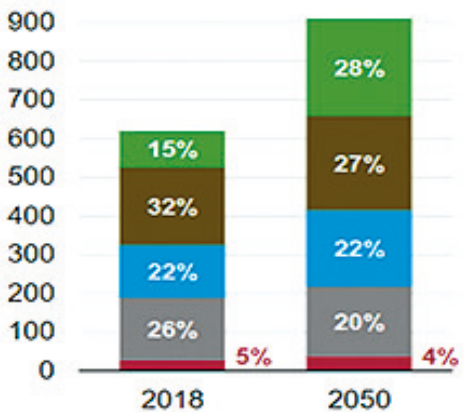

Imagen 1. Fuente: EIA (2019).

Algunas de las compañías más importantes, tradicionalmente productoras de hidrocarburos (petróleo y gas natural), también se han sumado a este fenómeno de la descarbonización del sector energético y se encuentran inmersas en la transición energética. Por ejemplo, REPSOL, empresa española con presencia en la región latinoamericana, recientemente ha publicado su plan estratégico

3 Según los datos publicados por Statista en el estudio "World carbon dioxide emissions in 2018, by sector" se tiene información de emisiones al 2018 de los siguientes sectores: electricidad y producción de calor, otros usos propios de la industria energética, manufactura y construcción, transporte, transporte por carretera, residencial, y comercial y servicios públicos. Si se suma el primero y el segundo, se puede apreciar que los sectores relacionados con la energía representan en total el 39.99\% del total de emisiones de carbono para el año 2018 (Statista, 2018). 
2021-2025 en el que señala su compromiso de alcanzar cero emisiones al 2050 con la implementación de metas intermedias de $10 \%$ al 2025, 20\% al 2030 y $40 \%$ al 2040, o el caso de Shell, que se encuentra comprometida a reducir la huella de carbono de sus productos vendidos en un 30\% para el 2035 y un 65\% antes del 2050.

Estas compañías, junto con British Petroleum, Total y otras empresas de gran envergadura del sector hidrocarburos, se encuentran apostando por diversificar sus negocios mediante la mejora de sus productos ${ }^{4}$ y la reducción de sus emisiones de carbono como consumidores (mejorando procesos, consumiendo energía limpia, etc.), así como proveedores (creando negocios bajos en carbono, inversión en actividades bajas en carbono, etc.).

En ese contexto, entre otros mecanismos para dar cumplimiento a los compromisos ambientales, se plantea el uso de impuestos para coadyuvar a que la neutralidad del sector energético sea una realidad o al menos, en defecto de lo anterior, se logre reducir gradualmente la emisión de GEI hasta alcanzar los compromisos establecidos por cada país en el marco del Acuerdo de París.

En ese sentido, desde la perspectiva impositiva varios autores han planteado el carácter extrafiscal ${ }^{5}$ de los tributos dentro de la solución regulatoria para la mitigación de externalidades negativas que afectan a la sociedad, como es el caso de la contaminación o el cambio climático, entre otros.

\section{APROVECHAMIENTO DE RECURSOS ENERGÉTICOS RENOVABLES (RER) EN EL PERÚ}

En el Perú aún existen pocos incentivos tributarios aplicables a la generación energética sustentable; sin embargo, antes de nombrar aquellos que ya existen dentro de la legislación peruana, es de vital importancia conocer con un poco más de profundidad los tipos de RER con potencialidad y cuáles son las fuentes que las crean. Así, es de precisar que las fuentes comúnmente usadas y estudiadas a lo largo del territorio nacional principalmente son: biomasa; eólica; geotérmica; mini hidráulica; solar; y mareomotriz y oleaje ${ }^{6}$.

4 Entre otros ejemplos, respecto de la mejora de productos, se puede mencionar el objetivo de la compañía British Petroleum para recortar la intensidad en carbono en sus productos al $50 \%$ para el año 2050.

5 Su finalidad no es la habitual recaudación de fondos estatales para el cumplimiento de los roles del Estado o las necesidades sociales, sino que su principal objetivo es la preservación del medio ambiente.

$6 \quad$ Nótese que la producción energética total para el 2020 fue de 49,178.88 GW, con una mayor participación de recursos hídricos (57\%) y el gas natural (37,6\%), para luego dar paso en 
En los términos acotados por la Oficina Regional para Centroamérica de Biomass Users Network (BUN-CA, 2002) en su manual sobre energías renovables, la biomasa se refiere a toda la materia orgánica que proviene de árboles, plantas y desechos de animales que pueden ser convertidos en energía; o las provenientes de la agricultura (residuos de maíz, café, arroz), del aserradero (podas, ramas, aserrín, cortezas) y de los residuos urbanos (aguas negras, basura orgánica y otros), siendo la fuente de energía renovable más antigua conocida por el ser humano. Los principales usos técnicos de la biomasa pueden encontrarse en la producción de calor y la producción de biogás o biocombustibles. En el Perú, este sector ha encontrado su nicho en la producción de energía mediante el uso del bagazo de la caña de azúcar, palma aceitera y la cascarilla de arroz principalmente. Para el año 2020, la participación en generación de energía mediante biogás y bagazo fue de 0,50\% del total anual.

Por su parte, la energía eólica hace referencia a aquellas tecnologías y aplicaciones en que se aprovecha la energía cinética del viento, convirtiéndola en energía eléctrica o mecánica mediante el uso de molinos o aerogeneradores. El primero de ellos se utiliza, fundamentalmente, para el bombeo mecánico de agua y los generadores que son equipos esencialmente diseñados para producir electricidad (Ministerio de Energía y Minería, 2008, p. 4). En 2016, el Ministerio de Energía y Minas (MEM) señaló que el potencial eólico del Perú era de aproximadamente 22,450 Megavatios (MW); sin embargo, en dicho año se estaban aprovechando $239 \mathrm{MW}$; es decir, únicamente un 1\% del potencial total. En 2020, se produjeron 1,803.20 MW de energía por fuente eólica, lo que representó $3,67 \%$ de la matriz energética peruana, demostrándose así un leve incremento, pero sin llegar a un porcentaje considerable del potencial eólico.

La energía geotérmica, según la definición adoptada por el Consejo Europeo de Energía Geotérmica (EGEC), es aquella energía almacenada en forma de calor por debajo de la superficie sólida de la tierra y engloba el calor almacenado en rocas, suelos y aguas subterráneas, cualquiera que sea su temperatura, profundidad y procedencia. Este tipo de energía es bastante antigua y existía incluso antes que el término fuera creado, siendo probablemente una de las

cantidades menores a recursos como el carbón (0.1\%), diésel y residuales $(0.3 \%)$, y los recursos energéticos renovables (RER) (eólicos $3.1 \%$, solar $1.4 \%$, bagazo $0.4 \%$ y biogás $0.1 \%$ ). Aunque la estadística muestra que la mayor parte de la energía proviene de recursos hídricos en general (convencionales y no convencionales), es pertinente señalar que, de ese total, únicamente se considera como parte de las RER de fuente hídrica a la energía producida por centrales hidroeléctricas con potencia instalada menor a $20 \mathrm{MW}$, comúnmente llamadas minihidráulicas, que tienen participación en la producción energética de 4.24\%. 
energías más antiguas que el ser humano haya podido conocer. Sin embargo, por la complejidad que conlleva la transferencia de calor y los procesos de obtención de energía no ha sido suficientemente desarrollada en el país (Llopis y Rodrigo, 2008, p. 25).

Este tipo de energía, según ha informado el Banco Mundial, podría representar un 3.5\% de la producción mundial de electricidad para el 2050 con 200 GW de capacidad instalada, evitando 760 millones de toneladas de emisiones de $\mathrm{CO}_{2}$ cada año. En el Perú, la energía geotérmica tiene un potencial de 3,000 MW y las regiones de mayor potencial geotérmico son Puno, Arequipa, Moquegua, Ayacucho, Puno y Pasco; no obstante, al 2020, se ha evidenciado una nula participación de la energía geotérmica en la producción de energía, según los datos publicados por el Comité de Operación Económica del Sistema Interconectado Nacional (COES).

Por otro lado, la energía generada mediante mini hidráulicas se refiere a la energía obtenida mediante el aprovechamiento de la energía cinética y potencial de la corriente de los ríos, saltos de agua o mareas; sin embargo, a diferencia de la energía hidráulica convencional, es decir, específicamente las centrales hidráulicas que cuentan con una potencia instalada menor a $20 \mathrm{MW}$, es un tipo de energía no convencional por su mínimo impacto ambiental y el uso de las aguas sin necesidad de represarlas. Al respecto, el Instituto para la Diversificación y Ahorro de Energía (IDAE) de España señala que la potencia instalada no constituye el criterio básico para diferenciar una minicentral de una central hidroeléctrica convencional, toda vez que, desde el punto de vista de la obra civil, una central obedece a principios completamente distintos a las grandes centrales alimentadas por enormes embalses, de tal forma que los trabajos de construcción son muy reducidos en comparación y sus impactos sobre el medio ambiente pueden ser minimizados si se desarrollan las medidas correctoras necesarias para ello (Castro, 2006, p. 26).

En relación con la energía solar, según la definición planteada por el IDAE, se la considera como aquella energía proveniente de la radiación solar que a su vez puede ser, según su aplicación eléctrica: solar fotovoltaica, por la cual la radiación solar llega a los nódulos, produciendo energía eléctrica por el efecto fotovoltaico, pudiendo almacenarse en baterías para su posterior consumo; $y$, solar térmica, por la cual se utiliza la radiación solar directa concentrada para el calentamiento de un fluido que, a su vez, se utilizará para producir vapor, accionando turbinas y finalmente generando energía eléctrica (IDAE, 2010, p. 27).

Según el Banco Mundial, el 93\% de la población mundial vive en países que tienen un potencial promedio diario de energía solar fotovoltaica entre 3 y 5 
kilovatios hora por kilovatio de capacidad instalada (kWh/kWp). En específico, Latinoamérica cuenta con Argentina, México, Chile y Perú como países que poseen excelentes condiciones para la energía solar (ESMAP, 2020, p. 9), tal como se muestra a continuación en el siguiente gráfico.

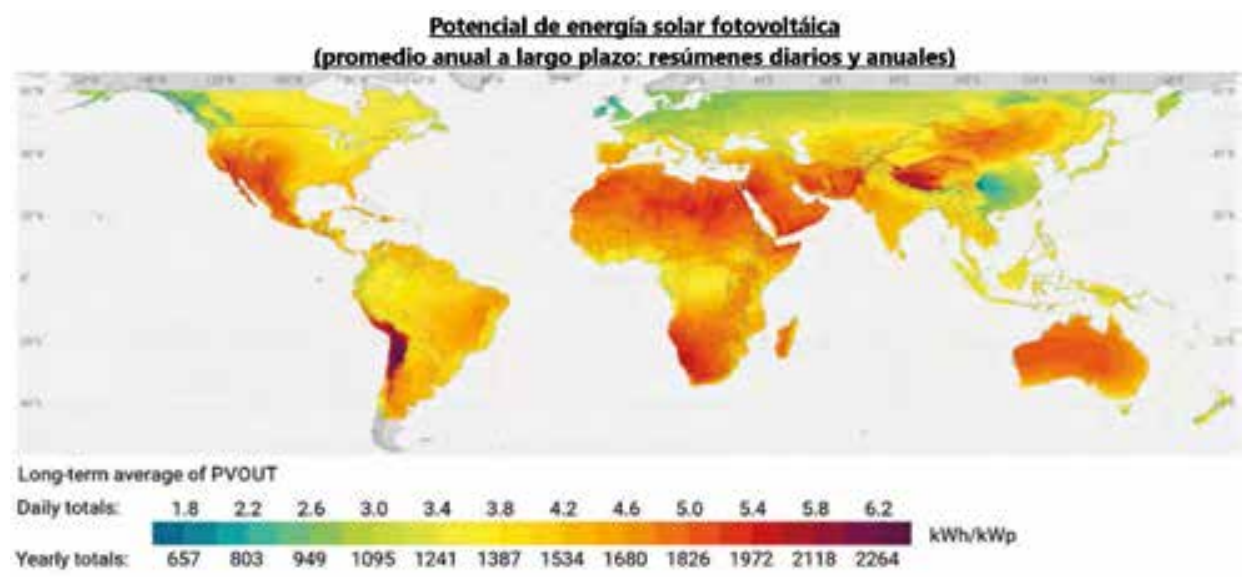

Imagen 2. Fuente: Banco Mundial (2020).

Finalmente, dentro de los recursos renovables se encuentra la energía proveniente del oleaje o mareomotriz que a su vez se puede clasificar por su utilización en: energía de las corrientes, en la que se hace uso de la energía cinética que poseen las corrientes marinas mediante la instalación submarina de estructuras similares a un aerogenerador; energía mareotérmica, que emplea la variación entre la temperatura de la superficie y la temperatura de aguas profundas; energía undimotriz o energía a partir del movimiento de las olas, que utiliza la fuerza con la que se desplaza determinada masa de agua a causa del rozamiento con las corrientes de aire; la potencia osmótica o también llamada energía azul, obtenida mediante osmosis por la diferencia de concentraciones de sal entre agua de mar y el agua de los ríos; y la energía mareomotriz, que aprovecha el ascenso y descenso de agua producidas por las fuerzas gravitatorias (Quintero y Quintero, 2015, p. 122).

Todas estas energías tienen en el mundo un potencial eléctrico que podría ser utilizado para cubrir necesidades energéticas con bajas o nulas emisiones de GEl, situación que ha despertado el interés de varios países en el desarrollo 
e implementación de políticas y tecnología para la producción de energía con RER; por ende, incorporan incentivos económicos, regulatorios, tributarios y otros que se comentan más adelante.

\section{BREVES COMENTARIOS SOBRE EL SECTOR ENERGÉTICO PERUANO, LA GENERACIÓN MEDIANTE RER Y SUS INCENTIVOS}

La energía es considerada como un bien esencial para el desarrollo de la sociedad y del ser humano, llegando a ser indispensable para muchas actividades cotidianas, otorgando una vida digna bajo la provisión de electricidad, gas y otros servicios regulados por el Estado; además, es de suma importancia para el desarrollo de industrias en todos los sectores económicos.

Al respecto, el Organismo Supervisor de la Inversión en Energía y Minería (OSINERGMIN, en adelante) ha precisado que la industria eléctrica ${ }^{7}$ es pieza clave para el desarrollo económico y social de un país, debido a que la electricidad es un insumo esencial para la producción de la mayor parte de los bienes y servicios de una economía. En los últimos 25 años, el Perú ha sido testigo de importantes transformaciones en la industria eléctrica (2016, p. 15).

Entonces, se parte del supuesto de que, sin energía eléctrica, la sociedad como se la conoce no sería la misma ni tendría el avance que se ha documentado en la historia. Sin embargo, en el afán de generar cada vez más electricidad para sostener la creciente demanda de esta, se han emitido miles de toneladas de $\mathrm{CO}_{2}$ en dicho proceso, llegando a emitir a nivel global 36,441 millones de $\mathrm{MtCO}_{2}$ al 2019 de las cuales el sector energía eléctrica y calórica representa el mayor porcentaje $^{8}$. Desde esa perspectiva, múltiples países alrededor del globo se encuentran comprometidos a promover una industria energética poco emisora.

Al respecto, se debe prestar especial importancia a la evolución de la demanda eléctrica que, en el caso del Perú (igual que en el resto de países del mundo), se ha ido incrementando constantemente desde los 3,590 MW en enero de 2007 a 7,318 MW en febrero de 2020, según los datos del Sistema

Nótese que la eléctrica es una de las muchas industrias del sector energético, por lo tanto, no debe confundirse uno con el otro pues existe una relación continente-contenido. Sin embargo, se advierte que durante el texto del presente artículo se hará mención al sector eléctrico, representando la producción de las diversas fuentes renovables o no, debido a que la medición que realizaremos sobra la base de la producción de cada fuente podría ser aquella traducida o utilizada en la industria eléctrica.

8 Al 2018 , el sector de la energía representó el $43.75 \%$ del total de emisiones para dicho año en el mundo. 
Eléctrico Interconectado Nacional (SEIN) y la producción que se ha visto incrementada desde los 2,235 GWH a los 4,397 GWH en los mismos periodos. Esta información resulta importante para determinar en qué medida las RER deberían tener participación en la matriz energética para poder cumplir las metas ambientales y diseñar las políticas o estrategias que finalmente favorecerían su mayor participación?

Otra variable importante de la que no se debe apartar la vista es la oferta energética, de la cual se puede precisar, según datos del Comité de Operación Económica del Sistema (COES, en adelante), que el total de producción de energía eléctrica de las empresas generadoras del COES al 2019 fue de 52,889.14 GW, un $4.08 \%$ superior a los resultados obtenidos en el 2018 , con una participación mayoritaria de la generación con recursos hídricos, sin embargo, aún se habría contado con bajas participaciones de las $\operatorname{RER}(2019$, p. 1).

Datos de los cuales se puede afirmar que, mientras mayor sea el desarrollo del país y conforme se avance en el tiempo, la demanda de energía seguirá incrementándose, razón por la cual, en el contexto global de cambio climático en el que el Perú se ha comprometido a reducir sus emisiones de Gases de Efecto Invernadero (GEI) en 30\% al 2030 mediante inversiones y gastos con recursos públicos y privados, y un 10\% adicional en la medida que el Perú acceda a financiamiento internacional ${ }^{10}$, corresponde al Gobierno y los diversos actores del sector energético priorizar la generación eléctrica mediante fuentes renovables que ayuden a reducir las emisiones de GEI y alcanzar las metas planteadas.

Ahora bien, con relación a la producción eléctrica mediante RER, en el 2019, habrían entrado en operación comercial siete centrales hidroeléctricas (la mayoría con potencia instalada menor a $20 \mathrm{MW}$ ) y dos termoeléctricas cuyo funcionamiento se encuentra basado en el uso de bagazo. La participación de los recursos renovables en la producción total de energía eléctrica del SEIN alcanzó el 8.52\%, un 1.29\% más que el 2018 evidenciando una tendencia al incremento de las energías renovables en la matriz energética peruana (COES,

9 Luego de una baja en la demanda en los meses de marzo y abril de 2020 a raíz de la emergencia sanitaria vivida en el mundo atribuida a los efectos del COVID-19, la tendencia fue al alza y de rápida recuperación, cerrando el 2020 con 7,125.30 MW y alcanzando en total un 1.54\% más que el año 2019.

10 Según el reporte de actualización de las Contribuciones Determinadas a Nivel Nacional del Perú (NDC por sus siglas en inglés), aprobado el 16 de diciembre de 2020 por la Comisión de Alto Nivel de Cambio Climático, la meta total del Perú sería en total del 40\%, aspirando a que las emisiones de gases de efecto invernadero alcancen un nivel máximo de 179,0 $\mathrm{MtCO}_{2}$ eq en el año 2030. 
2019, p. 5); sin embargo, a pesar del ligero incremento, aún resulta necesaria la mayor participación de las RER.

En el ámbito tributario, de los incentivos que contempla actualmente la legislación peruana para la generación de energía mediante recursos renovables resaltan:

i. El régimen de depreciación acelerada otorgado por el Decreto Legislativo $\mathrm{N}^{\circ} 1058$

Aplicable a las maquinarias, equipos y obras civiles necesarias para la instalación y operación de la central de generación de energía mediante uso de RER, que sean adquiridos a partir de julio de 2008. Esta norma establece una tasa de depreciación, a efectos de determinar el Impuesto a la Renta, no mayor a $20 \%$ como tasa global anual, beneficio que se encuentra vigente hasta el 31 de diciembre de 2025.

ii. La Ley Orgánica de Recursos Geotérmicos de 1997 (Ley N²6848).

Habilita la amortización de gastos incurridos en etapa de exploración en función de unidades de producción o mediante amortización lineal de tales gastos acumulados durante un periodo no menor a 5 años a partir del inicio de la producción.

En adición a lo comentado previamente, resulta conveniente mencionar que el Decreto Legislativo N 1002 estableció, como incentivo regulatorio a los generadores cuya producción se encuentra basada en RER, la prioridad en la conexión para el despacho y acceso a las redes eléctricas de transmisión y distribución, hasta el límite del 5\% del consumo nacional de electricidad. Asimismo, el mismo cuerpo normativo señaló que, en caso de que el costo marginal resulte menor a la tarifa determinada por el OSINERGMIN, el precio de la energía en el mercado sería complementado por una prima, considerando la clasificación de las instalaciones por categorías y grupos según las características de las RER.

Uno de los grandes desafíos para el sector energético peruano radica en la promoción e implementación de políticas dirigidas a que el crecimiento energético sea sustentable tanto de la oferta como de la demanda y con base en recursos que soporten la reducción de emisiones de GEI.

\section{INCENTIVOS FISCALES PARA ENERGÍAS RENOVABLES EN EL MUNDO}

En vista de que uno de los motores principales para el incentivo al uso de RER en la generación eléctrica son el cambio climático y el cumplimiento de las metas ambientales de cada país, las soluciones para estos problemas, en los 
temas impositivos, han sido bastante creativas e interesantes especialmente en países europeos, como Bélgica, España e Irlanda, que actualmente se encuentran incentivando la generación de energía eléctrica mediante RER para lograr la descarbonización de sus sectores energéticos.

Usualmente, tales impuestos son creados con carácter extrafiscal, ya que su finalidad principal no radica en la recaudación, sino en desincentivar el uso de energía cuya fuente sea contaminante frente a alternativas más ecológicas como las energías renovables; así, estos impuestos estarían relacionados con el principio básico ambiental "el que contamina, paga". Este principio, según comenta la Comisión Económica para América Latina y el Caribe (CEPAL), sería adoptado a nivel internacional por primera vez en 1972 por recomendación del Consejo de la Organización para la Cooperación y el Desarrollo Económicos (OCDE) sobre "principios directores relativos a los aspectos económicos internacionales de políticas ambientales" y hace referencia principalmente a que la persona natural o jurídica responsable de una contaminación, debe pagar los gastos de las medidas necesarias para evitarla o para reducirla (1991, p. 6).

La Directiva N ${ }^{\circ}$ 2004/35/CE del Parlamento Europeo, en su momento, definió claramente el principio comentado y añadió como definición de daño medioambiental a los daños que produzcan efectos significativos en el estado medioambiental (ecológico, químico o cuantitativo) de los recursos hídricos; los daños al suelo que supongan un riesgo significativo para la salud humana; los daños a las especies y hábitats naturales protegidos que ofrezcan efectos adversos en la conservación; y, la liberación de contaminantes a la atmósfera, en aguas inferiores superficiales y en aguas subterráneas, así como cualquier liberación intencional en el medio ambiente de organismos modificados genéticamente.

Los impuestos bajo este principio se aplican en diversas fases de la producción eléctrica, desde el desarrollo de nuevas tecnologías hasta el consumo de energía proveniente de fuentes contaminantes; no obstante, también existen en múltiples países incentivos fiscales cuya función es afianzar las inversiones y el uso de energía generada bajo fuentes renovables.

Al respecto, Ogunlana y Goryunova señalaron que múltiples países europeos se encontraban utilizando incentivos fiscales para promover la inversión y desarrollo de tecnología desde aproximadamente 1990 e iniciaron sus propios programas de incentivo a las energías alternativas y a la mejora de la eficiencia energética; resaltando, entre todos ellos, el crédito fiscal a las inversiones aplicable tanto en Estados Unidos como en los países europeos (2016).

Adicionalmente, en el caso de Estados Unidos se encuentran también incentivos tributarios relacionados con los proyectos con RER, tales como el 
crédito fiscal por producción de electricidad renovable (PTC), el crédito fiscal por inversión en determinados proyectos de energía renovable (Investment Tax Credit, ITC), el crédito fiscal por energía renovable en residencias y sistemas de depreciación acelerada. Adicionalmente, existen programas de subvenciones y préstamos impulsados por diferentes entidades del Gobierno que fomentan en gran medida la inversión, producción y uso de energía renovable.

En el siguiente cuadro comparativo se aprecia un breve resumen sobre los incentivos tributarios para energía alternativa entre Europa, Corea, China y Estados Unidos al 2016 y del cual se observa que la reducción de impuestos es ampliamente utilizada para promover las energías renovables.

\begin{tabular}{|l|l|l|l|}
\hline $\begin{array}{c}\text { Incentivos fiscales para inversiones en } \\
\text { energías alternativas en Europa, } \\
\text { Corea, China y EE. UU. }\end{array}$ & $\begin{array}{c}\text { Incentivos } \\
\text { Fiscales }\end{array}$ & $\begin{array}{c}\text { Tasa } \\
(\%)\end{array}$ & Tecnologías \\
\hline Bélgica & $\begin{array}{l}\text { Reducción } \\
\text { de impuestos }\end{array}$ & 14.5 & Todas \\
\hline España & Crédito fiscal & 12 & Todas \\
\hline Irlanda & $\begin{array}{l}\text { Reducción } \\
\text { de impuestos }\end{array}$ & 12.5 & Todas \\
\hline China & $\begin{array}{l}\text { Reducción } \\
\text { de impuestos }\end{array}$ & 15 & $\begin{array}{l}\text { Solar, Eólica, } \\
\text { Giomasa y }\end{array}$ \\
\hline Estados Unidos & Crédito fiscal & 30 & Solar \\
\hline Corea del Sur & Crédito fiscal & 10 & Todas \\
\hline
\end{tabular}

Cuadro 1. Fuente: Ogunlana y Goryunova (2016).

Al respecto, en el año 2014, KPMG Internacional (2015) preparó un informe comparativo en alrededor de 31 países sobre incentivos a las energías renovables bajo normas de promoción tanto regulatorias como fiscales, en el cual se aprecia que el Perú carece, principalmente, de créditos fiscales por inversión o producción con renovables en comparación con el resto de países, situación que aún hoy se mantiene. 
Por ejemplo, para el caso de Estados Unidos, el Investment Tax Credit (ITC) para energía solar se estableció en el año 2006 (30\% hasta el 2019) y, actualmente, se aplica como un crédito fiscal de $26 \%$ contra el impuesto a la renta para propietarios de sistemas solares residenciales o comerciales que podrán exigir el crédito mientras se encuentre vigente. Algunos de los gastos considerados para el crédito son: paneles solares o células fotovoltaicas; costos de mano de obra del contratista; equipamiento (cableado, inversores y equipo de montaje); dispositivos de almacenamiento; etc.

En España, además de los créditos fiscales, también existen bonificaciones para las imposiciones en los tributos locales (municipales), como en el impuesto sobre Bienes Inmuebles (IBI) y el Impuesto sobre Instalaciones, Construcciones y Obras (ICIO). Por aplicación de estos beneficios, se establecen bonificaciones en el IBI de hasta un 50\% del total del impuesto para los bienes inmuebles en los que se hayan instalado sistemas para el aprovechamiento térmico o eléctrico de la energía proveniente del sol. Por otro lado, en el ICIO, se establecen bonificaciones de hasta el 95\% para construcciones que incorporen energía solar.

Ahora bien, más allá del crédito fiscal aplicable al impuesto sobre la renta y tributos locales que determinados países han incluido en sus legislaciones, cabe resaltar que la herramienta de incentivo fiscal de mayor uso es el establecimiento de tasas bajas en el Impuesto al Valor Agregado (IVA) por kWh (similar al Impuesto General a las Ventas en el Perú), aplicando mecanismos como la devolución anticipada del IVA para inversiones en proyectos de generación con RER, exenciones del impuesto o rebaja de tasas para consumidores. En un contexto en el que el IVA representa una buena parte de los precios pagados por kWh, estas medidas representan un incentivo importante para los consumidores energéticos que podrían encontrar en la reducción de tasas o en la exención de impuestos incentivos económicos de ahorro en su día a día, prefiriendo en consecuencia el consumo de energía con fuentes renovables antes del consumo de la energía proveniente de contaminantes.

En Filipinas, existen exenciones fiscales para las importaciones de maquinaria, equipo y materiales para instalaciones de energía renovable por un plazo de 10 años mediante una certificación de un tercero desarrollador de energía renovable (Republic of the Philippines, 2008). Esta modalidad, establecer tasa cero para importaciones de insumos para energías renovables, también sería utilizada por diversos países como Brasil, Honduras o, recientemente, hasta en pequeñas repúblicas como Maldivas.

Nótese que, en el primer semestre del 2020, el IVA y el pago de otros impuestos representó alrededor del $41 \%$ del precio de la electricidad para 
consumidores domésticos medianos en Europa (entre 2,500 kW y 5,000 kW al mes), siendo los países con porcentajes más elevados Dinamarca con el $64 \%$, Alemania con el $54 \%$ y Portugal con el $49 \%$, hecho que eleva el interés por utilizar energías renovables que ayuden a rebajar los montos pagados en impuestos por el consumo energético.

\section{Electricity prices for household consumers, first half $\mathbf{2 0 2 0}$ (EUR per $\mathrm{kWh}$ )}

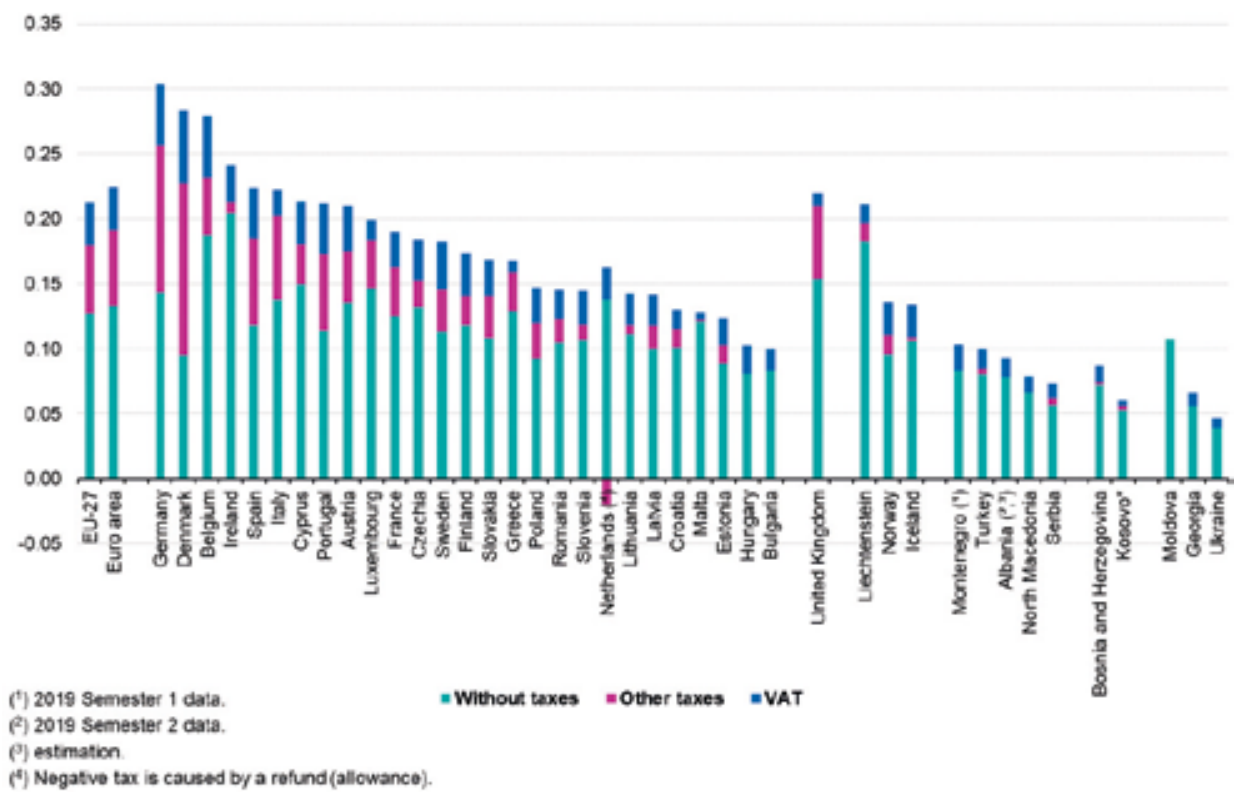

Imagen 3. Fuente: Eurostat (2020).

Esto demuestra que la participación de los impuestos en las inversiones energéticas juega un rol importante que debe ser evaluado frente a la implementación de otros mecanismos de fomento a las RER.

\section{EL IMPUESTO AL CARBONO}

Otro instrumento fiscal que viene siendo adoptado para la reducción de la emisión de GEl en diversos países y que se encuentra ligado al consumo de energía es el impuesto sobre el carbono (Carbon Tax), cuya finalidad principal es desincentivar el consumo de combustibles fósiles y otras fuentes energéticas 
contaminantes. Este impuesto normalmente se encuentra incluido en el precio de los combustibles y la energía eléctrica, es decir, finalmente termina siendo trasladado a los consumidores.

En el caso de la contaminación producida por las actividades privadas, determinadas como externalidades negativas por su concepción económica, este impuesto es recomendado para soslayar esta externalidad, intentar atenuar sus efectos y desincentivar el uso de energía contaminante según lo señalado por la OCDE y el Banco Mundial. Adicionalmente, se señala que un impuesto al carbono típico establece un enlace directo entre las emisiones de GEI (medidas en toneladas métricas de Dióxido de Carbono Equivalente o $\mathrm{tCO}_{2} \mathrm{e}$ ) de un producto o proceso y el impuesto que debe pagarse sobre ellas. Asimismo, para que el impuesto al carbono sea implementado exitosamente, previamente se deben realizar diversos pasos como entender el impuesto; realizar una comparación del impuesto con otros instrumentos de política fiscal; y evaluar varios instrumentos de política fiscal ante objetivos específicos (Alianza de Preparación para los Mercados del Carbono, 2017).

También por recomendación del Banco Mundial y la OCDE, la implementación del impuesto debería estar inspirada en los principios de equidad, alineación de las políticas y objetivos, estabilidad y previsibilidad, transparencia, eficiencia y rentabilidad, $y$, finalmente, de confiabilidad e integridad ambiental (Alianza de Preparación para los Mercados del Carbono, 2017).

Ahora bien, al 2020, más de 40 países en el mundo habían adoptado un impuesto al carbono o alguna otra modalidad de precio sobre el carbono como mecanismos de compensación, financiamiento climático o sistemas de comercio de emisiones, de los cuales Canadá es el país con el sistema más rígido respecto a su cumplimiento y ambiciones, cuya tasa al 2019 fue de US\$15 por tonelada de $\mathrm{CO}_{2}$ y se incrementará a US\$38 por tonelada de $\mathrm{CO}_{2}$ en el año 2022.

Por otro lado, en la región Sudamérica, Colombia, Argentina y Chile son los únicos países que cuentan con impuestos al carbono, mientras que Brasil aún no ha fijado posición al respecto, pero ya se encontraría evaluando su implementación, tal como se observa en el mapa elaborado por el Banco Mundial, el cual también muestra el avance de la implementación de mecanismos de precio al carbono. 


\section{Mapa de iniciativas regionales, nacionales y subnacionales de fijación de precios del carbono (2020)}

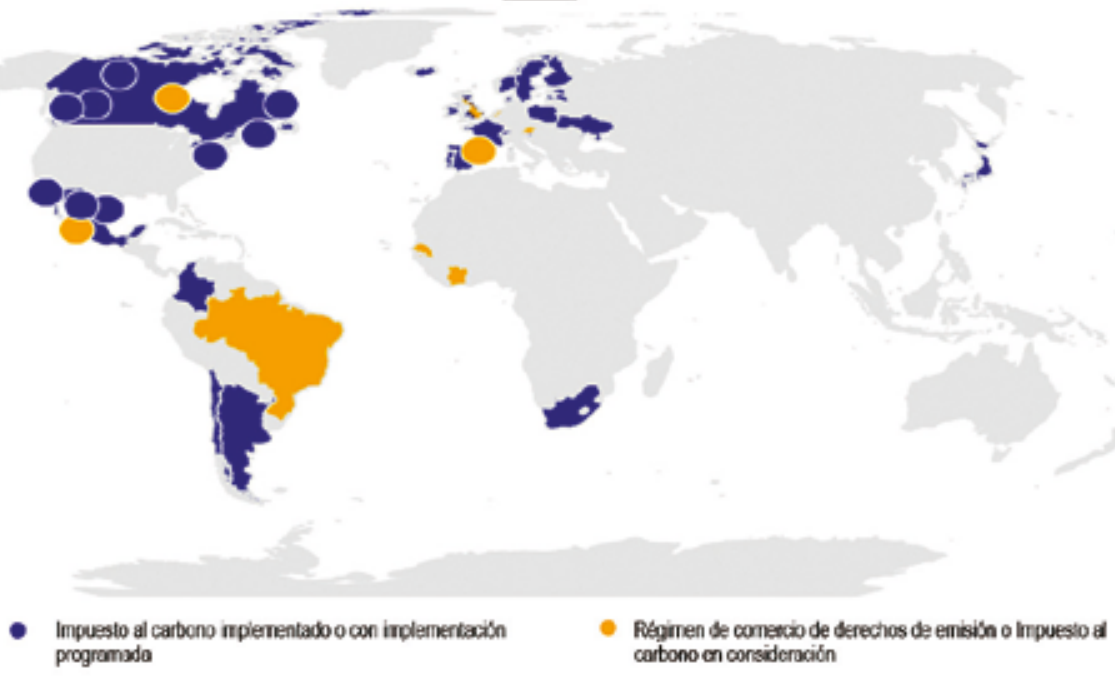

\section{Imagen 4. Fuente: Carbon Pricing Dashboard- Banco Mundial(2020).}

\subsection{Chile}

Se puede observar que el sistema chileno ya cuenta con impuestos al carbono desde la publicación de la Ley $\mathrm{N}^{\circ} 20780^{11}$, mediante la cual se introdujeron los siguientes impuestos:

- Impuesto a la primera venta de vehículos de acuerdo a sus emisiones de Óxidos de Nitrógeno $\left(\mathrm{NO}_{\mathrm{x}}\right)$ y su rendimiento urbano ${ }^{12}$.

- Impuesto a las fuentes fijas en relación con la emisión de contaminantes locales como Dióxido de Azufre $\left(\mathrm{SO}_{2}\right)$, Óxidos de Nitrógeno $\left(\mathrm{NO}_{x}\right)$ y Material Particulado (MP) que cuentan con calderas o turbinas que en conjunto

11 Cabe resaltar que la norma fue publicada en el año 2014; sin embargo, para efectos de la aplicación del impuesto, su vigencia se estableció a partir del 2017, situación que gatilló la primera recaudación por este tipo de impuestos recién en el ejercicio 2018.

12 Las disposiciones aplicables a este impuesto se encuentran contenidas en el artículo $3^{\circ}$ de la Ley $N^{\circ} 20780$. 
sumen una potencia térmica igual o superior a $50 \mathrm{MW}$ térmicos por establecimiento $^{13}$

- Impuesto directo a la emisión de Dióxido de Carbono $\left(\mathrm{CO}_{2}\right)$ aplicado a fuentes fijas ${ }^{14}$.

Corresponde mencionar que estos impuestos se crearon bajo el principio aguas abajo ${ }^{15}$ y son impuestos de difícil determinación por los componentes que contienen, toda vez que incluyen dentro de las fórmulas para la determinación de cada uno de ellos elementos como calidad del aire, costo social, población y otros.

En ese sentido, el gobierno chileno ha llegado a implementar una nueva infraestructura institucional más compleja que la tradicional, ya que incluye la participación del Ministerio del Ambiente de dicho país en la determinación del impuesto, precisamente, para que esta institución soporte al Servicio de Impuestos Internos (autoridad tributaria chilena) en la determinación e incorporación de los sujetos afectos al impuesto.

\subsection{Argentina}

En Argentina, se implementó el impuesto al carbono desde el año $2018^{16}$, mediante la aprobación de la Ley $\mathrm{N}^{\circ} 27430^{17}$ con un esquema un tanto más sencillo, pues tan solo se habrían añadido montos fijos en pesos para

13 Las disposiciones aplicables a este impuesto se encuentran contenidas en el artículo $8^{\circ}$ de la Ley $\mathrm{N}^{\circ} 20780$.

14 Las disposiciones aplicables a este impuesto se encuentran contenidas en el artículo $8^{\circ}$ de la Ley $N^{\circ} 20780$.

15 Para mayor entendimiento del término Aguas abajo es importante referirse a los comentarios de Huanca Ayaviri, quien precisa que un ejemplo clásico de externalidades negativas son la contaminación de ríos o lagos producidos por los extractores de minerales y otros ajenos a la práctica común como puede ser la pesca excesiva o medios no adecuados (dinamita, envenenamiento); como consecuencia los pescadores o agricultores que aprovechan las aguas río abajo ven reducida o afectada la pesca o la producción agrícola (Huanca, 2015, p. 112). En ese contexto, según explican Vasco, Tobón y Vargas, el término aguas abajo hace alusión a una externalidad negativa de tipo espacial, por el que los agentes económicos generalmente ignoran el impacto en los beneficios y el bienestar de los agentes ubicados en otro sitio. Ello implica en este caso que se graven las emisiones generadas, no el contenido de carbono de los combustibles fósiles utilizados (Vasco, C.A., Tobón, D. y Vargas, J.H., 2018, p. 596).

16 Previamente, Argentina no contaba con ningún esquema explícito de precio al carbono.

17 Las disposiciones aplicables al impuesto al carbono se encuentran en el artículo $139^{\circ}$ de la Ley $\mathrm{N}^{\circ} 27430$. 
determinados productos como nafta, gasolina, solventes, carbón mineral y otros, determinando como sujetos pasivos a aquellos importadores definitivos, productoras de petróleo y otros productores y elaboradores de carbón mineral.

Según Gutman, la tasa impositiva de este impuesto equivalía a USD 10/ $\mathrm{tCO}_{2}$ e de acuerdo con el tipo de cambio vigente al 1 de enero de 2018; $\sin$ embargo, por el proceso devaluatorio se fue reduciendo para llegar aproximadamente hasta los US\$ 6/tCO 2 e. Cabe resaltar que el proyecto original establecía una tasa impositiva de US\$25/ $\mathrm{tCO}_{2}$ e y había previsto destinar $25 \%$ de los ingresos recaudados por dicho impuesto a financiar medidas de transición energética. No obstante, el proyecto final habría sido aprobado precisando que el $100 \%$ de los ingresos generados serían distribuidos entre el Gobierno nacional y los Gobiernos subnacionales (Gutman, 2019, p. 89).

\subsection{Colombia}

En Colombia, también se viene aplicando el impuesto al carbono desde el año 2017, introducido por la Ley de Reforma Tributaria Estructural N 1819 de $2016^{18}$, por la que se ha dispuesto que el dinero recaudado con los instrumentos económicos verdes (impuestos ambientales) se destinen al Fondo para la Sostenibilidad Ambiental y Desarrollo Rural Sostenible en zonas afectadas por el conflicto, al manejo de la erosión costera, la conservación de fuente hídricas y la protección de ecosistemas.

Al igual que en el caso argentino, en Colombia se adoptó un mecanismo sencillo para el impuesto al carbono, añadiéndose determinados montos a la venta, retiro o importación de combustibles fósiles como la gasolina, keroseno, jet fuel, gas natural y otros, precisando que la tarifa asciende a 15000 pesos por tonelada de $\mathrm{CO}_{2}$.

Lo mencionado anteriormente, respecto de los tres países se resume en el siguiente cuadro en el que se indica desde cuándo es aplicable el impuesto al carbono y la tasa aproximada del impuesto aplicable al 2019 en dólares por

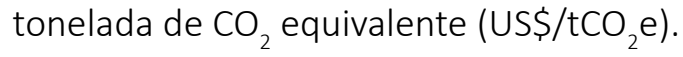

18 Nótese que las disposiciones aplicables al impuesto al carbono se encuentran en los artículos 221 a 223 de la Ley N 1819 de 2016. 


\section{Cuadro de Impuestos al Carbono en Sudamérica}

\begin{tabular}{|c|c|c|}
\hline País & $\begin{array}{c}\text { Tasa del Impuesto } \\
\text { (aproximado al 2020) }\end{array}$ & Vigencia \\
\hline Argentina & US\$ 6/tCO $\mathrm{e}$ & 2018 \\
\hline Chile & US\$ 5/tCO $\mathrm{e}$ & 2017 \\
\hline Colombia & US\$ 5/tCO $\mathrm{e}$ & 2017 \\
\hline
\end{tabular}

Cuadro 2. Fuente: Elaboración propia.

\section{REFORMA FISCAL MEDIOAMBIENTAL EN EL PERÚ}

Como ya se ha señalado, respecto de los incentivos a la generación con RER y la estructura del Impuesto al Carbono, en el Perú aún hay mucho por hacer. Esta situación resulta preocupante a todas luces, pues, si el Perú desea alcanzar su meta de reducir en un 40\% su emisión de GEl al 2030, se debe plantear una reforma del sector energético que incluya una reforma fiscal ambiental, incluso tomando el ejemplo de países como Argentina o Chile en los que la inclusión de sus impuestos al carbono conllevó paralelamente una reforma bastante importante en los sistemas tributarios de ambos países.

Dentro de la reforma ambiental, se sugiere eliminar los subsidios que incentiven actividades ambientales nocivas para el medio ambiente, gravar en mayor medida las fuentes contaminantes o incentivar la generación y consumo de energía renovable

Según lo precisado por la OCDE, existen demasiados usuarios de energía que no pagan los precios de la energía y del carbono necesarios para frenar el cambio climático, situación que podría desencadenar un incumplimiento casi completo de los objetivos del Acuerdo de París (2019).

Para el caso peruano, debido al incremento anual de demanda de energía que se viene sosteniendo desde 2007 y que, muy probablemente siga incrementando, diversos incentivos a la inversión, generación y consumo de energías renovables, incluyendo el análisis de si amerita incluir un impuesto al carbono, serían de gran ayuda para que dicha demanda se vea satisfecha en un futuro con energía generada con un mínimo de emisión de GEI.

Algunos esfuerzos ya se han venido haciendo en materia fiscal, pero faltaría un planeamiento estratégico mucho más profundo para incorporar, además de 
cambios regulatorios, una reforma fiscal. Los cambios más recientes en el Perú se han introducido en los Decretos Supremos Nº94-2018-EF y 306-2016-EF, respecto del Impuesto Selectivo al Consumo (ISC), elevando las tasas aplicables a los combustibles más contaminantes y reduciéndolas para aquellos que contaminen menos.

Ahora bien, otro cambio importante ha sido la modificación al ISC introducida por el Decreto Supremo $N^{\circ} 181-2019-E F$, en el que, si bien es cierto no se recoge un alza de la tasa aplicable para los combustibles en razón de su nivel de contaminación, sí se implementan tasas preferenciales para vehículos menos contaminantes de acuerdo al cilindraje del vehículo (a mayor cilindraje mayor tasa) y también se incorporan partidas arancelarias de vehículos a gas, híbridos y eléctricos usados en la lista de productos gravados con una tasa del $40 \%$, cuando anteriormente se encontraban gravados tan solo con un $10 \%$. Al respecto, cabe resaltar que en la actualidad los vehículos eléctricos nuevos están exentos del ISC (Decreto Supremo N095-2018-EF).

Todo ello indica que en el Perú el ISC es actualmente el impuesto más utilizado como medida para remediar las externalidades negativas producidas por el consumo de fuentes contaminantes; sin embargo, podría evaluarse otros mecanismos a fin de que se introduzcan en la política fiscal ambiental.

Nótese que, según ha precisado Mantilla (2020), recientemente la Comisión Multisectorial de Emisiones Vehiculares se encontraría evaluando la comercialización únicamente de dos tipos de gasolinas debido a la sobresaturación del mercado actual y la permanencia de combustibles contaminantes como los gasoholes de 84 octanos, 90 octanos.

Otro aspecto fundamental para evaluar en una futura reforma fiscal ambiental en el Perú es la capacidad de las instituciones públicas para desarrollar sinergias entre sí y poder implementar impuestos al carbono complejos como es el caso de Chile, o en su defecto, se diseñe de tal forma que se pueda alcanzar la finalidad propuesta y pueda ayudar realmente a que el Perú cumpla los compromisos establecidos en el Acuerdo de París.

\section{CONCLUSIONES}

Es evidente que, conforme la sociedad peruana siga desarrollándose, la demanda de energía se incrementará en el transcurso de los próximos años; en ese contexto, corresponde al Estado fomentar la inversión, producción y consumo de energía con bajas emisiones para que finalmente se pueda alcanzar la meta de reducción de GEI en un 30\% al 2030 y un 10\% adicional en la medida que el Perú pueda acceder a financiamiento internacional. 
En el escenario internacional existen múltiples países que actualmente se encuentran utilizando mecanismos de promoción fiscal para la generación con energías renovables menos contaminantes con muy bajas o cero emisiones.

La introducción del impuesto al carbono es una de las fórmulas utilizadas en materia fiscal y podría ser evaluada, ya que, en el Perú, actualmente las modificaciones y cambios han estado más vinculados con el ISC.

Finalmente, la inclusión de todos estos nuevos mecanismos a la legislación peruana ameritaría una reforma fiscal medioambiental que podría tomar como base las recomendaciones de la OCDE y la experiencia internacional, teniendo en cuenta la realidad peruana.

\section{LISTA DE REFERENCIAS}

Alianza de Preparación para los Mercados del Carbono (Partnership for Market Readiness, PMR). (2017). Guía del Impuesto al Carbono: Un Manual para Creadores de Política. Banco Mundial.

Banco Mundial. (2020a). Carbon Pricing Dashboard [imagen de archivo]. https:// carbonpricingdashboard.worldbank.org/

Banco Mundial. (2020b). Solar Photovoltaic Power Potential by Country [imagen de archivo]. https://www.worldbank.org/en/topic/energy/publication/solar-photovoltaic-power-potential-by-country

BUN-CA. (2002). Manuales sobre energía renovable-Biomasa. Fundación Red de Energía (BUN-CA).

Castro, A. (2006). Manuales de Energías Renovables- Minicentrales Hidroeléctricas. Instituto para la Diversificación y Ahorro de Energía.

CEPAL. (1991). El principio "El que contamina, paga". Revista de la CEPAL, 45, 7788. https://doi.org/10.18356/acc86f0a-es

COES. (2019). Informe de la operación anual del SEIN 2019.

EIA. (2019). TODAY IN ENERGY. EIA projects nearly 50\% increase in world energy usage by 2050, led by growth in Asia [infografía]. https://www.eia.gov/ todayinenergy/detail. php?id=41433

EIA. (2019, 24 de septiembre). TODAY IN ENERGY. EIA projects nearly 50\% increase in world energy usage by 2050, led by growth in Asia. Consultado el 13 de enero de 2021.

https://www.eia.gov/todayinenergy/detail.php?id=41433

ESMAP. (2020). Global Photovoltaic Power Potential by Country. World Bank. 
Eurostat. (2020). File: Electricity prices for household consumers, fist half 2020 (EUR per kWh) [infografía]. https://ec.europa.eu/eurostat/statistics-explained/ index.php?title=File:Electricity_prices_for_household_consumers,_first_ half_2020_(EUR_per_kWh).png\&oldid $=505000$

Fanelli, J. M., Jiménez, J. P. y López, I. (2015). La Reforma Fiscal Ambiental en América Latina. Naciones Unidas.

Gutman, V. (2019). Argentina: Descarbonización energética y precios al carbono. En C. Trinidad (Ed.), Precio al Carbono en América Latina: Tendencias y Oportunidades (pp. 56-107). Sociedad Peruana de Derecho Ambiental

Huanca, F. (2015). Estudio del Derecho desde la Economía. Revista Jurídica Derecho, 2(3), pp. 97-114.

IDAE. (2010). Guía práctica de la energía. Consumo eficiente y responsable. IDAE. KPMG International. (2015). Taxes and incentives for renewable energy. Expositivo, KPMG.

Llopis, G. y Rodrigo, V. (2008). Guía de la Energía Geotérmica. Fundación de la Energía de la Comunidad de Madrid.

Mantilla, J. (2020). Menos gasolinas, más beneficios para todos. Revistaenergía. pe, 21, pp. 87-90.

Ministerio de Energía y Minería. (2008). Energías Renovables 2008- Energía Eólica. Ministerio de Energía y Minería- Secretaría de Energía.

OCDE. (2019). Taxing Energy Use 2019: Using Taxes for Climate Action. OCDE Publishing.

Ogunlana, A. y Goryunova, N. (2016). Tax Incentives for Renewable Energy: The European Experience. EPSBS. https://doi.org/10.15405/epsbs.2017.01.69

OSINERGMIN. (2016). La industria de la electricidad en el Perú: 25 años de aportes al crecimiento económico del país. Editado por Jesús Francisco Roberto Tamayo Pacheco, Julio Salvador Jácome, Arturo Leonardo Vásquez Cordano y Carlo Magno Vilches Cevallos. OSINERGMIN.

Quintero, J. R. y Quintero, L. E. (2015). Energía mareomotriz: potencial energético y medio ambiente. Gestión y Ambiente (Universidad Nacional de Colombia) 18(2), pp. 121-134.

Republic of the Philippines. (16 de diciembre de 2008). Republic Act No. 9513.

Statista. (2018). World carbon dioxide emissions in 2018, by sector. https://www. statista.com/statistics/276480/world-carbon-dioxide-emissions-by-sector/

Vasco, C. A., Tobón, D. y Vargas, J. H. (2018). Impuestos Ambientales Diferenciados Espacialmente en Colombia: Un modelo teórico de equilibrio general con capital natural. Editado por Cuadernos de Economía 37. Universidad Nacional de Colombia. https://doi.org/10.15446/cuad.econ.v37n74.61553 